\title{
BELLEZA, PROBABILIDAD Y ADAPTACIÓN EN LA ESTÉTICA DE FRANCIS HUTCHESON
}

\author{
JORGE LÓPEZ LLORET \\ Universidad de Sevilla
}

\begin{abstract}
RESUMEN: El presente artículo propone una aproximación a la estética de Francis Hutcheson diferente a la tradicional, la cual se centraba en la identificación de la belleza como experiencia del sujeto y en la evaluación del impacto de este subjetivismo sobre la generación posterior. Como alternativa se acentúan los aspectos relacionales de su noción de belleza, en la que la estructura del objeto y la respuesta del sujeto no se pueden separar. Para ello el autor se centra especialmente en la poco estudiada Sección V del Tratado I de Una investigación sobre el origen de nuestras ideas de belleza y virtud. En ella la fórmula de la uniformidad en la diversidad, la matemática de la probabilidad y la adaptación de los seres vivos a sus condiciones de vida justifican la hipótesis de Dios diseñador, lo que sitúa a la belleza en el centro del pensamiento de Hutcheson y define una nueva aproximación a su influjo sobre las generaciones posteriores, sobre todo Adam Smith.
\end{abstract}

PALABRAS CLAVE: Francis Hutcheson; estética; belleza; teología natural; argumento del diseño; ilustración escocesa.

\section{Beauty, Probability and Adaptation in the Aesthetics of Francis Hutcheson}

ABSTRACT: This article proposes an approach to the aesthetics of Francis Hutcheson different from the traditional one, which centered in the identification of beauty as an experience of the subject and the evaluation of the impact of this subjectivism on the subsequent generation. As an alternative are accentuated the relational aspects of his notion of beauty, in which the structure of the object and the response of the subject cannot be separated. For this the author focuses especially on the little studied Section V of the Treatise I of An Inquiry into the Original of Our Ideas of Beauty and Virtue. In it the formula of uniformity amidst diversity, the mathematics of probability and the adaptation of living beings to their life conditions justify the hypothesis of God as designer, which places the beauty at the center of Hutcheson's thought and defines a new approach to his influence on after generations, especially Adam Smith.

KEY WORDS: Francis Hutcheson; aesthetics; beauty; natural theology; design argument; scottish enlightenment.

\section{INTRODUCCIÓN.}

Arthur Hermann decía que ninguna figura de la Ilustración se había tratado tan de pasada como Francis Hutcheson ${ }^{1}$, lo que se puede explicar por la impresionante talla intelectual de sus antecesores (Shaftesbury) y sus sucesores (David Hume o

1 Herman, A., The Scottish Enlightenment. The Scots' Invention of the Modern World, London, Fourth State, 2003, p. 414. En términos similares se expresó Brown, Michael, Francis Hutcheson in Dublin, 1719-1730, Dublín, Four Courts Press, 2002, pp. 9-10. 
Adam Smith). No obstante, al estudiarlo así perdemos de vista su riqueza y complejidad, pues la obra de Hutcheson fue un crisol de la Modernidad que tuvo como fundente a la experiencia estética de la belleza.

En el pensamiento de Hutcheson se aunaron el pasado pagano y cristiano con el presente empirista y racionalista; la estética, la ética y la política con la ciencia física; el materialismo corpuscular con la teleología del diseño inteligente y la religión con las matemáticas. En este contexto introdujo lo que se considera su mayor logro, la subjetividad de la persona empírica, que parecía reducir a su condición mundana los temas tradicionales de la filosofía, incluida la estética. La naturaleza vertebradora y sutil de este pensamiento, sin embargo, lo hace frágil, pues es muy fácil desmembrarlo, lo que exige del intérprete una lectura que parta del respeto a la coherencia de su síntesis.

La historia no ha hecho justicia a la complejidad del pensamiento de Hutcheson ni al papel nodular y fundamental de la experiencia estética en él, lo que hace problemática la estimación correcta de su influencia sobre generaciones posteriores, especialmente sobre Adam Smith. La dificultad para asumir su pensamiento como un todo trabado ha hecho preferible el estudio del influjo de cada parte sobre pensadores posteriores en disciplinas especializadas, lo que ha pasado sobre todo con el estudio de su estética, desvinculada de su teleología, su teología, su economía, su política y su ética. Esto condujo a fallos estratégicos de interpretación, como la generalizada minimización del valor de la Sección V del Tratado I de An Inquiry into the Original of Our Ideas of Beauty and Vitue (1725) (a partir de ahora citaremos esta obra como An Inquiry)2.

William Robert Scott, en un estudio clásico, desmembró diacrónicamente esta complejidad del pensamiento de Hutcheson en cuatro fases. De ellas, la primera giraba en torno a An Inquiry y la crítica a Hobbes y Mandeville (desarrollada en las cartas dirigidas al Dublin Journal) y se hallaba bajo la influencia de Shaftesbury y Cicerón; la tercera, por su parte, marcada por el System of Moral Philosophy (1755), estaba más influida por Aristóteles y el razonamiento teleológico ${ }^{3}$. Nosotros, sin embargo, creemos más necesario desarrollar una lectura sincrónica como la de James Moore, para quien el pensamiento de Hutcheson apenas varió con el tiempo, debiéndose las diferencias que Scott detectó al tipo de público al que se destinaban las obras: lectores generales en un caso y estudiantes de la Universidad de Glasgow

2 Pensamos en estudiosos ilustres, dispares en el tiempo y en metodología, como George Dickie y Francisco Mirabent. Este, por ejemplo, se refirió de pasada a «numerosos ejemplos físicos y matemáticos, que no es preciso reproducir aquí» (Mirabent, F., La estética inglesa del siglo XVIII, Barcelona, Editorial Cervantes, 1927, p. 233); tiempo después, Dickie extendió un manto aún más opaco sobre esta Sección: "Como puede observarse, en el bosquejo anterior no se menciona la quinta sección. Esta sección es la más larga del libro de Hutcheson y está dedicada exclusivamente a la prueba del diseño a favor de la existencia de Dios. Esta incursión en el campo de la teología no es esencial para una comprensión de su teoría del gusto» (DickIE, G., El siglo del gusto. La odisea filosófica del gusto en el siglo XVIII, Madrid, Visor, 2003, p. 22).

3 ScotT, W. R., Francis Hutcheson. His Life, Teaching and Position in the History of Philosophy (1900), New York, August M. Kelley, 1966, pp. 182-197 (primera fase) y 210-229 (tercera fase). 
en otro ${ }^{4}$. Sin embargo, disentimos de él al no considerar que los únicos problemas medulares afrontados por Hutcheson se conectasen con la estética, la ética y la psicología. Nos resistimos a dejar de lado la teología natural y la teleología, importantes para Hutcheson desde 1725 y sin las cuales no se puede comprender el papel jugado por la estética de la belleza en su sistema ${ }^{5}$.

El plan de trabajo que hemos adoptado en el estudio que sigue se centra en la recuperación de la Sección V del Tratado I de An Inquiry, dado que nos parece uno de los episodios más relevantes e integradores del pensamiento de Hutcheson ${ }^{6}$. No creemos exagerar si afirmamos que contiene el argumento central de su obra. Por otra parte, aunque no es el objeto de este artículo (que pretende, no obstante, servir de base para eso, de lo que presentamos un ejemplo en la conclusión), a partir de aquí se podría redefinir la posición histórica de Hutcheson, sobre todo la influencia que ejerció sobre sus seguidores y críticos y, a través de ellos, sobre nosotros. En cualquier caso, se le hace más justicia a su legado, sobre todo en lo que se refiere a la estética.

\section{LA FÓRMULA DE LA BELLEZA}

En 1725 el irlandés Francis Hutcheson publicó en Londres An Inquiry, cuya cuarta edición, con variaciones, apareció en la misma ciudad en 1738. Fue uno de los textos básicos de la ilustración escocesa temprana, cuyo Tratado I se considera un hito fundacional de la estética moderna. El autor distinguió aquí dos tipos de belleza: original (o absoluta) y relativa (o comparativa ${ }^{7}$. La segunda es la que posee un objeto que imita a otro (que podría no ser bello), mientras que la primera es propia de un objeto considerado en sí mismo, según la siguiente definición:

The Figures which excite in us the Ideas of Beauty, seem to be those in which there is Uniformity amidst Variety [...] what we call Beautiful in Objects, to speak in the Mathematical Style, seems to be in a compound Ratio of Uniformity and

4 Moore, J., «The Two Systems of Francis Hutcheson: On the Origins of The Scottish Enlightenment», en: Stewart, M. A. (ed.), Studies in the Philosophy of Scottish Enlightenment, Oxford, Clarendon Press, 1990, pp. 41-42.

5 Véase Harris, J. A., "Religion in Hutcheson's Moral Philosophy», en: Journal of the History of Philosophy, 46/2 (2008), 205-222. Tal y como afirmó Hermann, su intención fundamental, nunca perdida de vista, fue «to change the face of theology in Scotland» (HermanN, A., The Scottish Enlightenment, p. 78); quien mejor ha indicado el papel de la teología en la estética de Hutcheson ha sido, en polémica con Kivy, SzécsénYI, Endre, «Francis Hutcheson and the Emerging Aesthetic Experience», en: Journal of Scottish Thought, 7 (2016), 171-209, especialmente pp. 180-189 y 206-209.

6 A partir de aquí, siempre que nos refiramos a esta parte de la obra de Hutcheson diremos, simplemente, Sección V, sin más indicación. Debe sobreentenderse que se ubica en el Tratado I de An Inquiry.

7 Hutcheson, F., An Inquiry into the Original of Our Ideas of Beauty and Virtue, Indianapolis, Liberty Fund, 2008. Se trata de la segunda edición, de 1726, si bien los editores recogen las variantes textuales de las otras tres. Sobre la belleza original o absoluta pp. 28-35; sobre la belleza relativa o comparativa pp. 42-45 (hay traducción castellana: Una investigación sobre el origen de nuestra idea de belleza, Madrid, Tecnos, 1992). 
Variety: so that where the Uniformity of Bodies is equal, the Beauty is as the Variety; and where the Variety is equal, the Beauty is as the Uniformity ${ }^{8}$.

Esta fórmula no era en sí misma original. En el contexto histórico de Hutcheson fue usada por autores de raigambre racionalista como Leibniz y, sobre todo, Crousaz, aunque estos no recurrieron a su «estilo matemático» ${ }^{9}$. Esta fórmula no es, pues, la que se ha usado para definir a Hutcheson como el padre de la estética moderna, sino su identificación de un sentido estético específico que remite la experiencia de la belleza al sujeto, pasando el objeto (y la idea de la belleza) a un segundo plano. Con esto estaba introduciendo un programa de investigación basado en la explicación de todos los fenómenos humanos a partir de nuestra condición mundana ${ }^{10}$. Según este proyecto la experiencia estética se explicaba a partir de la hipótesis de un sentido interno humano (un séptimo sentido) que respondía ante objetos que presentaban una combinación adecuada de uniformidad y variedad, pero no a partir de los objetos mismos.

Con todo, nosotros creemos que la estética no le interesaba a Hutcheson (a diferencia de sus analistas) como un ámbito de estudio aislado, lo que le llevo a conceder al menos la misma importancia a la definición de la belleza del objeto. Por eso queremos profundizar en su «estilo matemático», en su defensa de una razón compuesta de uniformidad más variedad como criterio objetivo de belleza. Como gran parte de los autores posteriores a Newton (entre ellos algunos de sus profesores y compañeros en Glasgow ${ }^{11}$ ), Hutcheson se tomó en serio el uso de las matemáticas en las ciencias humanas ${ }^{12}$. Sirvan como ejemplos destacados los apartados XI y XII de la Sección III del Tratado II de An Inquiry, donde proponía un

8 Hutcheson, An Inquiry, pp. 28-29.

9 Crousaz usó la fórmula en el Capítulo 3 de su Traité du Beau, de 1715 (Crousaz, J. P. de, Traité du Beau, París, Fayard, 1985, pp. 29-33). Véase Franzini, Elio, La estética del siglo XVIII, Madrid, Visor, 2000, pp. 91-98 y 109.

10 Herman, A., The Scottish Enlightenment, p. 63.

11 Pensamos en Gershom Carmichael, su profesor de Filosofía Moral en Glasgow, al que Hutcheson sucedió en 1730. Según Arthur Herman, fue uno de los primeros docentes escoceses en discutir la obra de Newton en sus clases (Herman, A., The Scottish Enlightenment, pp. 68-69). En sus Philosophical Theses de 1707, de hecho, se refirió al newtonianismo en términos próximos a Richard Bentley (Carmichael, Gershom, Natural Rights on the Threshold of the Scottish Enligtenment, Indianapolis, Liberty Fund, 2002, pp. 374-376).

12 CREMASChI, S., "La herencia newtoniana en la economía política del siglo XVIII», en Elena, Alberto, Javier Ordóñez y Mariano Colubi (Comps.), Después de Newton: ciencia y sociedad durante la Primera Revolución Industrial, Barcelona, Anthropos, 1998, pp. 70-101, especialmente p. 77. Un panorama de la ciencia y sus conexiones con la ilustración en Glasgow en la época de Hutcheson puede consultarse en Emerson, R. L., y Wood, P., «Science and Enlightenment in Glasgow, 1690-1802», en Withers, Ch. W. J., y Wood, P., Science and Medicine in the Scottish Enlightenment, East Linton, Tuckwell, 2002, pp. 79-141; un producto de la cultura científica de Glasgow, estrictamente contemporáneo de Hutcheson, fue Colin Maclaurin, uno de los primeros newtonianos escoceses. Véase Grabiner, J. V., «Maclaurin and Newton: The Newtonian Style and the Autority of Mathematics», en Withers, Ch. W. J., y Wood, P., Science and Medicine in the Scottish Enlightenment, pp. 143-170. 
método cuantitativo para computar la moralidad de una acción ${ }^{13}$, o, casi de más interés para nuestra interpretación (pues remite a un caso clásico de análisis matemático), las referencias introducidas en 1726 en la Sección V a la probabilidad de obtener un premio en el juego de la lotería ${ }^{14}$. Por otra parte, para Hutcheson las matemáticas eran hermosas en sí mismas y dotaban de belleza a cualquier teoría que las acogiera, como cuando en la Sección III del Tratado I se refirió a la belleza de los teoremas, poniendo como modelos para las ciencias humanas a Euclides y a Newton:

Such is Gravitation, in Sir Isaac Newton's Scheme; such also is the Knowledge of the Original of Rights, perfect and imperfect, and external; alienable and unalienable, with their manner of Translation; from whence the greatest Part of moral Dutys may be deduc'd in the various Relations of human Life ${ }^{15}$.

La relevancia estética de este «estilo matemático» se desarrolló sobre todo en la Sección V, cuyo título es «Concerning our Reasonings about Design and Wisdom in the Cause, from the Beauty or Regularity of Effects» ${ }^{16}$. Con esto Hutcheson quería fundamentar la prueba de la existencia y naturaleza de Dios que se conoce como «argumento del diseño». Aunque en su historia de este tema Jantzen sitúa el de Hutcheson en un capítulo dedicado a argumentos «inusuales» ${ }^{17}$, nosotros no lo consideramos tal, dada su coherencia con diversas líneas de investigación de su

13 Hutcheson, F., An Inquiry, pp. 128-132 (este aparato computacional se eliminó en la cuarta edición en 1738); el mismo planteamiento aparece en 1728 en sus Illustrations on the Moral Sense, en Hutcheson, F., An Essay on the Nature and Conduct of the Passions and Affections, with Illustrations on the Moral Sense, Indianapolis, Liberty Fund, 2002, p. 189 (también lo eliminó en la tercera edición de esta obra). Según el editor del segundo libro citado (p. 189, n. 55), trataba de poner en práctica el proyecto de matematización de la moral que Richard Cumberland anunció en De Legibus Naturae (1672), I, 5-9 (hemos consultado la traducción inglesa A Treatise of the Laws of Nature, Indianápolis, Liberty Fund, 2005, pp. 293-302); véase Aatto, S. K., y Brooks, G. P., «The Rise and Fall of Moral Algebra: Francis Hutcheson and the Mathematization of Psychology», Journal of the History of Behavioural Science, 17 (1981), 343-356 y BRown, M., «The Aesthetic of Political Economy: The Case of Francis Hutcheson», Journal of Scottish Thought, 7 (2016), 136-147.

14 Hutcheson, F., An Inquiry, p. 56.

15 Hutcheson, F., An Inquiry, p. 38 (la referencia a los derechos y a la vida civil fueron eliminados en la cuarta edición). Es históricamente muy interesante esta conexión de la teoría de Newton con el principio de la belleza y su posible extensión a las ciencias humanas. No es un tema muy estudiado por los expertos en Hutcheson, pero aparece con fuerza, en los términos genéricos empleados por nuestro autor, en la Historia de la Astronomía de Adam Smith, en cuyo caso sí que ha dado lugar a un amplio y complejo debate interpretativo. Véase Sмiтн, A., Essay on Philosophical Subjects, Indianapolis, Liberty Fund, 1982, pp. 97-105 (hay traducción castellana: Ensayos filosóficos, Madrid, Pirámide, 1998); como ejemplo del debate que ha suscitado este tema en Adam Smith, véase Montes LiRA, L., «La Influencia de Newton en Adam Smith», Anuario Filosófico, XLII/1 (2009), 137-158 o Schliesser, E., «Some Principles of Adam Smith's Newtonian Methods in the Wealth of Nations», Research in the History of Economic Thought and Methodology, 23/1 (2005), 33-74 (remitimos a las conclusiones del presente artículo).

16 Hutcheson, F., An Inquiry, p. 46.

17 Jantzen, B. C., An Introduction to Design Arguments, Cambridge, Cambridge University Press, 2014, pp. 97-98. 
época. George Dickie reconoció en parte esto cuando situó la estética de Hutcheson en el contexto cristiano de justificación de un Dios benevolente:

This kind of teleological-theological theorizing is thought very clearly, for example, in the theory of taste of the eighteenth-century philosopher Francis Hutcheson. Hutcheson claims that the innate mechanism that for him make up the faculty of taste was created by a benevolent deity to provide to human beings with pleasure in their lives ${ }^{18}$.

El horizonte cristiano trazado por Dickie es pertinente en el caso de Hutcheson, pero al ser tan genérico da un tono conservador a su pensamiento que no le corresponde. Por otra parte, Dickie es parcial al proyectar a Hutcheson sólo sobre el horizonte cristiano porque este argumento también se enraizaba en la antigüedad pagana (en concreto, en el argumento estoico presentado por Cicerón en De la naturaleza de los dioses), vinculándose además con pensadores modernos de cristianismo heterodoxo, como Shaftesbury (especialmente con su obra Los Moralistas). Tampoco es correcta la proyección sólo hacia atrás de la teología de Hutcheson, pues la misma se conecta con la vanguardia científica y filosófica de su momento, especialmente con una serie de disciplinas y tendencias sin las cuales no es posible comprender los dos siglos posteriores, como el cálculo de probabilidades o la adaptación de los seres vivos a sus condiciones de existencia. Era una doble vía, pues si los teólogos naturales trataban de aprovechar, a su manera, la ciencia de vanguardia, los científicos recurrían a los teólogos naturales para ubicar sus hallazgos en ámbitos socialmente aceptables ${ }^{19}$. En el punto de encuentro de estas cuatro vías (la cristiana y la pagana, la conservadora y la innovadora) se sitúa su definición de la belleza como uniformidad en la variedad.

Según Roger Scruton, Hutcheson nunca explicó en qué consistían la unidad y la variedad ${ }^{20}$. Nuestra opinión es que de haber analizado con cierto detalle la Sección V no hubiera llegado a dicha conclusión. Es lo que deseamos mostrar en lo que sigue.

\section{El PAPEL DE LA PROBABILIDAD EN LA DEFINICIÓN DE LA BELLEZA}

Cuando Hutcheson publicó An Inquiry el debate sobre las relaciones entre el azar, el orden y la intención (divina) en la constitución de la realidad era de rigurosa actualidad, no sólo como problema teológico y metafísico, sino también matemático, dado que en él se inmiscuía la naciente teoría de la probabilidad ${ }^{21}$.

18 Dickie, G., Art and Value, Oxford, Blackwell, 2001, p. 4.

19 La complejidad de estas relaciones fue muy bien tratada por MAYR, E., The Growth of Biological Thought. Diversity, Evolution, and Inheritance, Cambridge/Mass, The Belknap Press, 1982, pp. 91-112.

20 Scruton, R., Beauty. A Very Short Introduction, Oxford, Oxford University Press, 2011, p. 162.

21 Para una panorámica general véase Heyde, C.C., y Seneta, E. (Eds.), Statisticians of the Centuries, New York, Springer, 2001, pp. 23-64. 
El nacimiento del cálculo de probabilidades se sitúa a mediados del siglo XVII, con la correspondencia entre Pascal y Fermat ${ }^{22}$, considerándose plenamente constituido en la época de Laplace. Aunque éste lo desvinculó de la teología natural, previamente fue utilizado para demostrar que no fue el azar, sino Dios, el agente del orden en el mundo ${ }^{23}$. Antes de 1725 resultaron relevantes las aportaciones de Chrystiaan Huygens, Jakob y Nicolaus Bernoulli, Pierre Rémon de Montmort, Abraham De Moivre o John Arbuthnot ${ }^{24}$, casi todos conectados con la ciencia y la filosofía británicas, especialmente a través de la Royal Society. Su interés principal era matematizar la realidad social para lograr una certeza similar a la de la teoría newtoniana, aunque también en la física y la astronomía se utilizaron estos argumentos con el mismo deseo de demostrar la existencia de un diseñador de la realidad, la cual no podía deberse al azar ${ }^{25}$. Así lo utilizó, por ejemplo, Richard Bentley (asesorado por Newton) en su influyente A Confutation of Atheism ${ }^{26}$. Es difícil definir el grado de conocimiento que Hutcheson tuvo de esto, aunque eran conocidos su afición y dominio de las matemáticas y de la filosofía natural en la época de gestación de An Inquiry ${ }^{27}$. Para él, que asumió la crítica de Cicerón al atomismo y rechazó la génesis cartesiana de los torbellinos, la naciente matemática de la probabilidad era bastante útil.

Hutcheson conocía bastante bien este argumento de Sobre la naturaleza de los dioses:

No entiendo cómo quien estima que esto [la génesis de la realidad propuesta por el atomismo] ha podido ocurrir no piensa también que, si se reunieran en alguna parte innumerables réplicas de nuestras veintiuna letras [...] podrían

${ }^{22}$ Cierta influencia de la teoría de la probabilidad de Pascal le pudo llegar indirectamente a Hutcheson a través de Arnauld y Nicole, dada la conexión con aquél de la parte final de la lógica de Port Royal, que Hutcheson conoció y usó. Arnauld, A., y Nicole, P., La logique ou l'art de penser (1662), París, Gallimard, 1992, pp. 331-334.

${ }_{23}$ Sobre esta dimensión de las matemáticas en aquella época se han publicado varias obras, comenzando por la fundacional de Todhunter, I., A History of the Mathematical Theory of Probability from the Time of Pascal to that of Laplace (1865), New York, Chelsea Publishing, 1965, pp. 7-21 (sobre la correspondencia entre Pascal y Fermat) y 464-613 (sobre Laplace), hasta llegar a Hacking, I., The Emergence of Probability, Cambridge, Cambridge University Press, 2006, pp. 166-175.

24 Todhunter, I., A History of the Mathematical Theory of Probability, pp. 22-25 (Huygens), 56-77 (Jakob Bernoulli), 78-134 (Montmort), 135-193 (De Moivre), 194-199 (Nicolas Bernoulli y Arbuthnot); y David, F. N., Games, Gods and Gambling, Londres, Charles Griffin, 1962, pp. 70-97 (Pascal y Fermat), 110-122 (Huygens), 130-139 (Jakob Bernoulli), 140-160 (Montmort), 161-178 (De Moivre).

${ }_{25}$ Véase el estudio de la «moralización de las matemáticas» en Lorraine, D., Classical Probability in Enlightenment, Princeton/New Jersey, Princeton University Press, 1995, pp. 296-368.

26 Jantzen, B. C., An Introduction to Design Arguments, p. 65; Bentley, Richard, A Confutation of Atheism from the Origin and Frame of the World, Part II (1693), en CoHEN, I. B., Isaac Newton's Papers and Letters on Natural Philosophy, Cambridge/Mass., Harvard University Press, 1978, pp. 315-352; véase la Parte Primera de Hurlblutt III, RoBert H., Hume, Newton and the Design Argument, Lincoln, University of Nebraska Press, 1965, pp. 3-92.

27 Sсотт, W. R., Francis Hutcheson, pp. 15 y 19. 
formarse con ellas, al ser arrojadas a tierra, los Anales de Enio, de modo que pudieran leerse de seguido ${ }^{28}$.

Este fue el desencadenante de la Sección V, donde Hutcheson pretendía demostrar el diseño como causa, rechazando la posibilidad de que lo fuera el azar. Su diferencia con Cicerón residía en el hecho de que Hutcheson contaba con la nueva matemática de la probabilidad, que daba más peso al argumento.

Para comprender, a partir de ahí, la Sección V hay que tener en cuenta la naturaleza a priori y negativa del argumento, que era probabilístico, pero no estadístico (mientras que el argumento de la adaptación, que trataremos en el siguiente apartado, era estadístico y no probabilístico), dada su dependencia de la hipótesis genética del atomismo clásico ${ }^{29}$. Es a priori porque a posteriori es evidente que existe el orden ${ }^{30}$, pero si tratásemos de dar cuenta de la génesis de éste a partir del encuentro azaroso de un número indeterminado de partes que chocan al azar, sería mucho más esperable el desorden. Es negativo porque lo que demuestra, puesto que el orden existe, es que la causa no puede ser el azar. Pensemos esto como si fuese un juego de dados. La probabilidad a priori de sacar un as en una tirada de dados es de 1/6; la de obtener dos ases seguidos en dos tiradas consecutivas 1/36; la de diez ases en diez tiradas, 1/60466176. Si de facto siempre obtuviéramos un as sospecharíamos que un ser inteligente ha trucado el dado. $\mathrm{O}$ vayamos al terreno de Cicerón: si tenemos una bolsa con las veintisiete letras de nuestro alfabeto (y una bola en blanco, más las de los signos de puntuación, que no consideraremos) y extraemos una pieza (que se reintegraría de inmediato a la bolsa), la posibilidad de sacar «e» es 1/28; la posibilidad de obtener sucesivamente «en un lugar de la mancha de cuyo nombre no quiero acordarme», $1 / 28^{59}$ (es asombroso pensar en el Quijote completo). Sospecharíamos de nuevo que fue un ser inteligente quien seleccionó intencionadamente las letras (al menos si partimos de la hipótesis, que era la única que Hutcheson podía aceptar, de la duración limitada de nuestro mundo ${ }^{31}$, pues si fuera infinita tendríamos la certeza de que el Quijote se obtendría infinitas veces). Quien «trucaba» o seleccionaba las formas del mundo era, para Hutcheson, Dios. Pensemos por ejemplo en esta afirmación:

28 Cicerón, M. T., Sobre la naturaleza de los dioses, Madrid, Gredos, 1999, p. 233.

29 Véase Van Melsen, A., From Atoms to Atom. The History of the Concept of Atom, New York, Harper and Row, 1960, pp. 81-128. Aparte de Hobbes, Gassendi, etc., Hutcheson se basaba en referentes clásicos, especialmente la hipótesis epicúrea expuesta, y refutada, en Sobre la naturaleza de los dioses de Cicerón (pp. 113-114).

30 Según Peter Kivy, Hutcheson no utilizó ninguna demostración a priori de la existencia de Dios, pues en general aborrecía el argumento apriorístico. Por eso se centró en las pruebas de diseño en el mundo real que remitían a un diseñador inteligente, consideradas pruebas a posteriori. KIvY, P., The Seventh Sense. Francis Hutcheson and Eighteenth-Century British Aesthetics, Oxford, Oxford University Press, 2003, pp. 111-112. No obstante, el argumento probabilístico sólo tiene sentido a priori y como refutación, a la luz de la nueva matemática, de la parte genética de la teoría atomista, basada en el azar. Sobre esta base resultaba deseable buscar a posteriori pruebas de diseño.

31 Hutcheson, F., Logic, Metaphysics, and the Natural Sociability of Mankind, Indianapolis, Liberty Fund, 2006, p. 160. 
There is also the Same Probability, that in any one System of Matter an Undirected Force will produce a regular Form, as any one given irregular one, of the same degree of Complication: But still the irregular Forms into which any System may be rang'd, surpass in multitude the Regular, as Infinite does Unity; for what holds in one small System will hold in a Thousand, a Million, a Universe, with more Advantage, viz. that the irregular Forms possible infinitely surpass the Regular $^{32}$.

El argumento era estadísticamente impecable. Su premisa era que por cada forma regular (por ejemplo, un hexágono) pueden darse infinitas formas irregulares (figuras posibles de seis lados diferentes), aunque las formas naturales, como los copos de nieve o las celdas de las abejas, eran siempre regulares. Si pasamos de la geometría de las figuras regulares a sistemas más amplios, como una planta, un animal, un ecosistema o un sistema solar (que serían como libros compuestos por letras), el cálculo negaría crecientemente la probabilidad de obtener esas formas a partir del azar, lo que condujo a Hutcheson a la siguiente conclusión:

We see then how infinitely improbable it is, «that all the Powers of Chance in infinite Matter, agitated thro infinite Ages, could ever effect this small Composition of a Prism entering a Prismatick Bore; and, that all our hazard for it would at most be but as three is to the third Power of Infinite» ${ }^{33}$.

La definición de la belleza como razón compuesta de uniformidad y variedad tenía su razón de ser en el cálculo de probabilidades, como queda claro si atendemos a los ejemplos de Hutcheson en la Sección II del Tratado I: un triángulo equilátero es menos bello que un cuadrado, éste menos que un pentágono y éste menos que un hexágono porque "the Variety increases the Beauty in equal Uniformity» "34; por otra parte, un triángulo equilátero es más bello que uno isósceles y éste más que uno escaleno porque "The greater Uniformity increases the Beauty amidst equal Variety» ${ }^{35}$. Dickie consideró el razonamiento tras este ejemplo poco intuitivo (mucha gente preferiría el triángulo) y defectuoso (pues «los lados desiguales del triángulo escaleno le hacen ser más variado» $)^{36}$. Creemos que en este juicio influyó su decisión de no analizar la Sección $\mathrm{V}^{37}$, que nosotros consideramos esencial, lo que hace que este ejemplo nos resulte coherente con una red argumental asentada en el cálculo de probabilidades.

Por limitarnos al triángulo, creemos (difiriendo de Dickie) que en la interpretación del ejemplo no se deben introducir los ángulos ni la magnitud de los lados, dado que la definición de triángulo que manejaba Hutcheson era la que Euclides proporcionó en la Definición 19 del Libro Primero de sus Elementos: figura comprendida por tres rectas, nada más ${ }^{38}$; ahora bien, en un plano hay infinitas líneas rectas y cuando tres de ellas se cruzan se genera un triángulo. Hay que partir de aquí para asumir el ejemplo teniendo en cuenta la Sección V y la matemática de

\footnotetext{
32 Hutcheson, F., An Inquiry, p. 47.

33 Hutcheson, F., An Inquiry, p. 51.

34 Hutcheson, F., An Inquiry, p. 29.

35 Hutcheson, F., An Inquiry, p. 29.

36 Dickie, G., El siglo del gusto, pp. 39-40.

37 Dickie, G., El siglo del gusto, p. 22.

38 Euclides, Elementos, Libros I-V, Madrid, Gredos, 1991, p. 195.
} 
la probabilidad. Según esto, un cuadrado es más bello que un triángulo porque es más improbable, dado que entre el infinito número de líneas que existen en un plano es más complejo coordinar cuatro que tres; puesto que el cuadrado posee una variable más, es más improbable (como lo es obtener cuatro veces seguidas cara al tirar una moneda al aire en lugar de tres). Además, el triángulo equilátero es más bello que el isósceles también al ser más improbable, pues entre los triángulos que se pueden formar con tres líneas rectas en un plano hay infinitos isósceles posibles, pero sólo uno equilátero (es como si tuviéramos un dado con cinco doses y un as: sería más improbable obtener éste en una tirada). La fórmula de la uniformidad más la variedad da de sí todo su significado cuando ambos extremos del ejemplo se componen. Si todas las caras de un dado fueran as (pura unidad), que en un lanzamiento obtuviéramos as no significaría nada, como tampoco que con un dado normal obtuviéramos cualquier número entre el uno y el seis (pura diversidad). Conseguir la mayor uniformidad partiendo de la mayor diversidad era lo más improbable de todo.

Tanto la variedad como la uniformidad fueron valoradas por Hutcheson a causa de su improbabilidad sólo teniendo en cuenta la razón compuesta entre ellas, no porque todo esto resultara valioso en sí mismo sino porque demostraba negativamente que la realidad natural (en la que se daba razón compuesta de diversidad y uniformidad máximas) no podía ser efecto del azar sino de la dirección inteligente. Es decir, Dios trucaba los dados o escribía libros. Todo esto se corroboraba constantemente a posteriori cuando se observaba la adaptación de los seres vivos a sus condiciones de existencia. El cálculo de probabilidades, por lo tanto, mediaba entre la fórmula de la belleza y la evidencia del diseño o, más bien, era lo que tenían en común.

Si no excluimos de nuestro análisis la Sección V, la conclusión explícita es la altísima improbabilidad de que una fuerza no dirigida produzca una máquina tan compleja como el más simple animal, mucho más como todo un sistema del mun$\mathrm{do}^{39}$. Pero a posteriori se muestra que estas máquinas existen, como vamos a ver a continuación.

\section{La BELlEZA DE LAS ADAPTACIONES}

Una máquina compleja es una coordinación de partes numerosas que colaboran en el desarrollo de un mismo trabajo y, por eso, concuerda con la fórmula de la belleza de Hutcheson. Las máquinas complejas, referentes culturales prestigiosos desde la revolución científica del siglo XVII ${ }^{40}$, eran hermosas para nuestro autor, lo que hace que su estética se vincule más con la ingeniería que con el arte ${ }^{41}$.

Esto nos lleva al argumento a posteriori de Hutcheson, complementario del anterior: la adaptación de los organismos a sus condiciones de vida, ilustración incontestable del argumento global del diseño. La teoría de la probabilidad se estaba

39 Hutcheson, F., An Inquiry, p. 51.

40 Véase Westfall, R. S., The Construction of Modern Science: Mechanism and Mechanics, New York, John Wiley and Sons, 1971; sobre la conexión con la biología pp. 82-104.

41 Sсотт, W. R., Francis Hutcheson, p. 191. 
formando en 1725, pero la Teología Natural ya tenía sus textos canónicos y una presencia masiva en la cultura británica ${ }^{42}$, viviendo su época dorada ${ }^{43}$. Hutcheson, educado para la iglesia y progresista, estuvo expuesto a esa manera de afrontar el hecho religioso por sus compatriotas científicamente formados. En Glasgow fue alumno de John Simpson y del citado Carmichael, quienes habían favorecido la teología natural por encima de la revelación ${ }^{44}$. Además, conocía las obras más relevantes de esta corriente, como The Wisdom of God manifested in the Works of the Creation (1691) de John Ray, la Physico-Theology (1713) y la Astro-Theology (1714) de William Derham ${ }^{45}$, o The Religious Philosopher (1715) de Bernard Nieuwentyt, traducida al inglés por John Chamberlayne ${ }^{46}$. Finalmente, defendía la universalidad del argumento del diseño elaborado por Shaftesbury ${ }^{47}$. Era, por otra parte, una literatura a la que la emergente matemática de la probabilidad, que buscaba negar el potencial genético del azar, no era ajena ${ }^{48}$.

42 Historiadas relativamente pronto por Hicks, E. L., A Critique of Design Arguments. A Historical Review and Free Examination of the Methods of Reasoning in Natural Theology, New York, Charles Scribner's Sons, 1883; en pp. 133-207 trata la época que gira en torno a Hutcheson, aunque no considera la obra de éste.

43 Jantzen, B. C., An Introduction to Design Arguments, p. 59. Jantzen estudia la época en pp. 58-98. Sobre el «argumento del diseño» en general véase McPherson, T., The Argument from Design, London, Macmillan, 1972 y De Cruz, H., y SMEDT, J. de A Natural History of Natural Theology, Cambridge/Mass., MIT Press, 2015, pp. 61-84; sobre la religión racional en el contexto de Hutcheson aconsejamos StEwART, M. A., "Religion and Rational Theology», en Broadie, A., The Cambridge Companion to the Scottish Enlightenment, Cambridge, Cambridge University Press, 2010, pp. 31-59 (en p. 39 se refiere al significado teológico de la belleza).

44 Herman, A., The Scottish Enlightenment, pp. 66-68 y 76. Hutcheson y Carmichael reconocieron mutuamente sus vínculos intelectuales. Pensemos en la obra del segundo titulada A Sinopsis of Natural Theology; aunque publicada en 1729, y aunque mostrando conocimiento y aprecio de las obras previas de Hutcheson, la sinopsis se propone como «suitable for the use of students», lo que remite a prácticas anteriores. Véase CARMichael, G., Natural Rights on the Threshold of the Scottish Enligtenment, p. 221.

45 Es interesante y relevante que Derham publicara en 1696 una obra titulada Artificial Clock-maker, dada la metáfora casi popular del Dios diseñador como relojero, desde Newton hasta Paley. El desarrollo de la metáfora del reloj en esa época ha sido bien tratado por MAYR, O., Autority, Liberty and Automatic Machinery in Early Modern Europe, Baltimore, The Johns Hopkins University Press, 1986, pp. 54-101.

46 Hutcheson se refería explícitamente a todos estos autores en la tercera edición de $A$ Synopsis of Metaphysics, en Hutcheson, F., Logic, Metaphysics and the Natural Sociability of Mankind, p. 153.

47 En el Tratado V de Characteristics, titulado The Moralist. A Philosophical Rhapsody, especialmente en la Sección IV de la Parte II (Shaftesbury, Anthony Ashley Cooper, 3er Conde de, Los Moralistas, Barcelona, Ediciones Internacionales Universitarias, 1997, pp. 160-183). Véase Arregui, J. V., «La Teleología de la Belleza en Shaftesbury y Hutcheson», Themata, Revista de Filosofía, 13 (1995), 11-35.

48 Por ejemplo, Arbuthnot, J., «An Argument for Divine Providence, taken from the Constant Regularity observed in the Births of both Sexes», en: Philosophical Transactions of the Royal Society of London 27 (1710), 186-190, cit. p. 186; véase Pearson, K., The History of Statistics in the 17th and 18th Centuries against the Changing Background of Intellectual, Scientific and Religious Thought, Londres, Charles Griffin, 1978, pp. 279-347 y BarThOLOMEw, D. J., God of Chance, Londres, SCM, 1984, pp. 38-40. 
Hutcheson introdujo en la Sección II del Tratado I de An Inquiry consideraciones que hacían de puente entre el argumento probabilístico y el de la adaptación, pues mostraban la geometría regular de los seres vivos. Vale la pena citar un ejemplo completo:

If we descend to the minuter Works of Nature, what vast Uniformity among all the Species of Plants and Vegetables in the manner of their Growth and Propagation! what exact Resemblance among all the Plants of the Same Species, whose Numbers surpass our Imagination! And Uniformity is not only observable in the Form in gross; nay, in this it is not so very exact in all Instances, but in the Structure of their minutest Parts, which no Eye unassisted with Glasses can Discern. In the almost infinite Multitude of Leaves, Fruit, Seed, Flowers of any one Species, we often see an exact Uniformity in the Structure and Situation of the smallest Fibres. This is the Beauty which charms an ingenious Botanist. Nay, what vast Uniformity and Regularity of Figure is found in each particular Plant, Leaf, or Flower! In all Trees and most of the smaller Plants, the Stalks or Trunks are either Cylinders nearly, or regular Prisms; the Branches similar to their several Trunks, arising at nearly regular Distances, when no Accidents retard their natural Growth: In one Species the Branches arise in Pairs on the opposite Sides; the perpendicular Plain of Direction of the immediately superior Pair, intersecting the Plain of Direction of the inferior, nearly at right Angles: In another Species, the Branches spring singly, and alternately, all around in nearly equal Distances: and the Branches in other Species sprout all in Knots around the Trunk, one for each Year. And in every Species, all the Branches in the first Shoots preserve the same Angles with their Trunk; and they again sprout out into smaller Branches exactly after the Manner of their Trunks. Nor ought we to pass over that great Unity of Colours which we often see in all the Flowers of the same Plant or Tree, and often of a whole Species; and their exact Agreement in many shaded Transitions into opposite Colours, in which all the Flowers of the same Plants generally agree, nay often all the Flowers of a Species ${ }^{49}$.

Aunque podríamos pensar en adaptaciones, aquí Hutcheson habla sólo de geometría regular, de relaciones numéricas y espaciales puras, es decir, de belleza absoluta, aunque encarnada en el mundo vegetal, uniforme en su diversidad y tan improbable que desafiaba a la imaginación. Si volvemos a la Sección V nos abrumará pensar el tema en términos de probabilidad y comprenderemos y sentiremos que quería mostrar que esto no podía ser obra de la casualidad. Hutcheson no nos permite pensar ni sentir que el azar pueda explicarlo, a diferencia del diseño ${ }^{50}$. La estructura lógica del razonamiento fue quizá más clara en A Synopsis of Metaphysics:

49 Hutcheson, F., An Inquiry, pp. 31-32. Recordamos que John Ray, uno de los principales artífices de la Teología Natural británica, fue igualmente uno de los botánicos más significativos de los siglos XVII y XVIII, con numerosas publicaciones desde que en 1660 publicó su Catalogue of Cambridge Plants hasta su obra Methodus Plantarum Emendata et Aucta, de 1703 (véanse los Capítulos IV y VIII-XI de Raven, Ch., John Ray, Naturalist: His Life and Works, Cambridge, Cambridge University Press, 2009, pp. 72-110 y 181-307). Seguramente parte de sus obras estaba tras este párrafo de Hutcheson.

50 Hutcheson, F., An Inquiry, p. 51. 
[...] all contingent things are effected either by necessary or by free causes. Furthermore, necessary causes which seem to act without design or will should be regarded as instruments which perform a function rather than acting, and when they do seem to be acting or impelling, they themselves are also acted upon or impelled. Only deliberately acting things, therefore, seem to have a real power within themselves, or truly to effect anything ${ }^{51}$.

Lo siguiente era introducir las adaptaciones, pues si se consideraba que esa recurrencia de la uniformidad en la diversidad (existiendo una infinita probabilidad de diversidad y desorden) producía la mejor vida posible para cada ser así ordenado, la necesidad de asentir lógicamente ante la presencia del orden como prueba del diseño se convierte en admiración y amor hacia el Creador ${ }^{52}$. La estética de la uniformidad en la diversidad conducía, así, de la geometría a la Fitness y de ésta a Dios a través del cálculo de probabilidades.

Hutcheson analizó la Fitness (y la idea del diseño, a la que conduce) en el ámbito de belleza relativa ${ }^{53}$. La geometría orgánica que organiza la forma adaptada genera belleza absoluta, pero el bienestar que esa forma permite alcanzar es algo derivado y, por lo tanto, relativo. La consideración de esta adaptación, dice Hutcheson, abre

[...] a new Scene of Beauty in the Works of Nature, by considering how the Mechanism of the various Parts known to us, seems adapted to the Perfection of that Part, and yet in Subordination to the Good of the Greatest whole, or of all Beings, to have been the Intention of the Autor of Nature ${ }^{54}$.

Esta adaptación a sus condiciones de vida armoniza la viabilidad y felicidad de los individuos con el bien del todo y, con ello, anticipa la fórmula de la ética utilitarista (el valor moral de una acción depende de la mayor cantidad de bienestar producida en el mayor número posible de seres), la cual parece guiar al Hacedor benigno y omnipotente, tal y como mostraban las obras de Ray, Derham o Nieuwentyt.

En la parte final de la Sección V Hutcheson le dio al argumento su forma clásica, es decir, analógica: si interpretamos los seres vivos adaptados como mecanismos y, por tanto, los asimilamos a las máquinas que nosotros diseñamos, puesto que éstas deben su existencia a nuestra inteligencia previsora, con aquellos pasará lo mismo ${ }^{55}$. Hutcheson comenzó en el Parágrafo X de la Sección V con un ejemplo simple (las ruedas de un carruaje), en el Parágrafo XVIII recurrió a máquinas complejas y finalmente, en el XIX, llegó a la imagen del Reloj, la cual ${ }^{56}$, proveniente de

51 Hutcheson, F., Logic, Metaphysics and the Natural Sociability of Mankind, p. 97.

52 Véase BRADY, E., "Hutcheson and Reid on Natural Beauty», Journal of Scottish Thought, 7 (2016), 29-51, especialmente pp. 34-38.

53 Hutcheson, F., An Inquiry, p. 34.

54 Hutcheson, F., An Inquiry, p. 45. Hay quien considera que, en última instancia, Hutcheson redujo la belleza relativa a la belleza absoluta, como Dickie, George, El siglo del gusto, p. 37.

55 La estructura lógica de este argumento, conocido como "argumento analógico», la presenta Jantzen, B. C., An Introduction to Design Arguments, p. 40. JanTZEN lo remite a Sobre la naturaleza de los dioses de Cicerón, un autor (y una obra) conocido y admirado por Hutcheson.

56 Hutcheson, F., An Inquiry, pp. 52, 57-58 y 58, respectivamente. 
Cicerón, fue la más común en el siglo XVIII desde que Nieuwentyt le diera su forma clásica en el Prefacio de The Religious Philosopher ${ }^{57}$.

Como podemos comprobar, Hutcheson estaba en el centro de los debates que eran de actualidad en el primer tercio del siglo XVIII. Ninguno de los argumentos que utilizó era nuevo por separado, pero de su unión surgió algo netamente original e influyente sobre el pensamiento de esa centuria, comenzando por la obra posterior del propio Hutcheson, cimentada en este primer Tratado estético. Con la uniformidad en la diversidad estableció un marco genérico en el que tenía cabida la noción de máquina, cuya definición se extendió a todos los seres vivos y de estos al conjunto del sistema newtoniano; finalmente, el recurso a la naciente teoría de la probabilidad concedía seguridad lógica al argumento. Por eso consideramos incorrecto calificar el deísmo de Hutcheson como contrario al racionalismo, dado que es una manera muy simple de etiquetarlo ${ }^{58}$. A la luz de la evidencia enumerada más arriba, que no se refiere a la moral de Hutcheson sino a su estética, podemos comprender que la emotividad y la racionalidad no se oponen sino, al menos en su caso, todo lo contrario. Además, si conectamos la fórmula de la belleza, mediante la matemática de la probabilidad, con la evidencia de las adaptaciones, resulta sorprendente la usual calificación de dicha fórmula como abstracta o vacía ${ }^{59}$, pues más bien se manifiesta plena de contenido referencial, un cuadro con el que enmarcar la realidad antes de someterla a un análisis (científico) más riguroso ${ }^{60}$.

Puesto que hemos nacido después de Hume, Darwin, Monod o Dawkins, no creemos demasiado en un Diseñador inteligente y benigno del mundo que nos rodea, lo que da lugar a que esta parte de los argumentos de Hutcheson nos interese menos que su hallazgo de la subjetividad estética, la identificación de un sentido de la belleza que lo condujo a la hipótesis de un «Internal Sense», a partir del cual derivaría la convicción religiosa ${ }^{61}$. Pero es un caso más de interpretación parcial de la evolución del pensamiento de Hutcheson, del papel y justificación del «Internal Sense» en el mismo y de su influencia sobre los pensadores de la generación inmediatamente posterior a la suya.

57 Nieuwentyt, B., The Religious Philosopher, Or, The Right Use of Contemplating the Works of the Creator, Vol. I. London, J. Senex / E. Taylor / J. Innys / J. Osborne, 1724 (tercera edición), pp. XXV-XXVII; Hicks, Lewis Ezra, A Critique of Design-Arguments, pp. 187-195.

58 CRowe, B. D., "Hutcheson on Natural Religion», British Journal for the History of Philosophy, 19/4 (2011), 711-740, cit. pág. 716.

59 Así lo propusieron, por ejemplo, GuYER, P., «Eighteenth Century Aesthetics», en DAvies, Stephen, Kathleen Marie Higgins, Robert Hopkins, Robert Stecker y David E. Cooper (eds.), A Companion to Aesthetics, Oxford, Blackwell, 2009, p. 44; Broadie, Alexander, «Art and Aesthetic Theory», en BroAdie, Alexander (ed.), The Cambridge Companion to the Scottish Enlightenment, Cambridge, Cambridge University Press, 2003, p. 282; esta opinión la revisa y matiza GÁRDos, Bálint, "“What could a Statue or Panegyrick effect?" - A Note on the Abstractness of Hutcheson's Aesthetics», Journal of Scottish Thought, 7 (2016), 15-28.

${ }_{60}$ La génesis concreta de la teoría estética de Hutcheson se opone al calificativo de abstracta, pues según Brown el origen de la fórmula de la belleza se debe conectar con las relaciones de Hutcheson y Molesworth en la época en la que éste estaba reformando su jardín en Brackdenstown, que sirvió de inspiración a aquél. Brown, M., Francis Hutcheson in Dublin, 1719-1730, pp. 47-50.

61 Crowe, B. D., «Hutcheson on Natural Religion», p. 729. 


\section{LA CONCEPCIÓN RELACIONAL DE LA BELLEZA}

La parte más estudiada de la estética de Hutcheson ha sido su dimensión subjetiva, sobre todo gracias a la magnífica obra de Peter Kivy The Seventh Sense. A éste no le importaron demasiado los argumentos de Hutcheson en torno al diseño, la improbabilidad genética del azar, la metáfora de la máquina o la adaptación, centrando su atención en sus vínculos con la epistemología de Locke. Así, la fórmula de la belleza le interesó más por la diferenciación de cualidades en primarias y secundarias, la teoría de la percepción y los significados del término «idea» que por los referentes contextuales e históricos de la experiencia estética que hemos identificado en el apartado anterior ${ }^{62}$.

Esta interpretación de Hutcheson es correcta, aunque de nuevo parcial cuando observa que su aportación a la historia de la estética consiste en haberla reducido a la naturaleza humana ${ }^{63}$. Esto proviene del estudio que hizo Jerome Stolnitz de la decadencia de la belleza objetiva en el siglo XVIII británico ${ }^{64}$. Según éste, el empirismo británico propuso una interpretación emocional de las tradicionales cualidades objetivas de la belleza ${ }^{65}$, produciéndose con Hutcheson una revolución copernicana en la estética, pues instaló al perceptor en el centro de las cosas, siendo su respuesta la que determinaba si un objeto era o no bello ${ }^{66}$. Esta interpretación, que se extendió al análisis de su teoría moral ${ }^{67}$, identificó el desinterés y la autonomía estética como las aportaciones más valiosas de Hutcheson, culminando con la Crítica del Discernimiento de Kant, lo cual, creemos, implica una lectura excesivamente teleológica de la historia de la estética ${ }^{68}$. Recogiendo una afirmación secundaria de Stolnitz, nosotros proponemos que el debate en torno al realismo o

62 KIVy, P., The Seventh Sense, pp. 260-265. Con todo, el tema del influjo de la teoría del conocimiento del Essay de Locke es complejo y ambiguo. Véase STOLNITZ, J., "Locke and the Categories of Value in Eighteenth Century British Aesthetic Theory», Philosophy, 38/143 (1963), 40-51; Townsend, D., "Lockean Aesthetics», en: The Journal of Aesthetics and Art Criticism, 49/4 (1991), 349-361, especialmente pp. 350-353, y KIVY, P., «Hutcheson's Idea of Beauty: Simple or Complex?», en: The Journal of Aesthetics and Art Criticism, 50/3 (1992), 243-245.

63 Fue el planteamiento de Arregui, J. V., «La Teleología de la Belleza en Shaftesbury y Hutcheson», en: Thémata, Revista de Filosofía, 13 (1995), 11-35 y, del mismo (bastante más matizado y equilibrado en sus planteamientos), «El Presunto Realismo Estético de Hutcheson», Thémata, Revista de Filosofía, 10 (1992), 629-657.

64 Stolnitz, J., "Beauty": Some Stages in the History of an Idea", en: Journal of the History of Ideas, 22/2 (1961), 185-204.

65 Stolnitz, J., "Beauty": Some Stages in the History of an Idea», p. 195.

66 Stolnitz, J., "Beauty": Some Stages in the History of an Idea», p. 201. Poco después Wladislaw Tatarkiewicz consagró definitivamente esta interpretación en Historia de seis ideas (1976), Madrid, Tecnos, 1990, pp. 170 y 247.

67 Este debate, en el contexto de la moral de Hutcheson, se produjo ante todo en los años ochenta del siglo pasado, por ejemplo, con la confrontación entre WiNKLER, K., «Hutcheson's Alleged Realism», en: Journal of the History of Philosophy, 23/2 (1985), 179-194 y NorTON, D. F., "Hutcheson's Moral Realism», Journal of the History of Philosophy, 23/3 (1985), 397-418.

68 Una presentación clásica de la lectura teleológica de la (experiencia) estética del siglo XVIII, donde se define el papel de precursor de Hutcheson, fue la de TownsEND, D., «From Shaftesbury to Kant: The Development of the Concept of Aesthetic Experience», en: Journal of the History of Ideas, 48/2 (1987), 287-305. 
subjetivismo de Hutcheson es innecesario y sólo conduce a visiones sesgadas de su modelo estético ${ }^{69}$.

La mejor introducción a la naturaleza compleja, imposible de someter a una sola etiqueta histórica, de los planteamientos de Hutcheson la proporcionó George Dickie, que sin embargo cometió el error estratégico de dejar fuera de sus consideraciones la Sección V, lo que tuvo notables consecuencias en la interpretación posterior de la estética de Hutcheson, sobre todo el establecimiento del «Internal Sense», el «Sense of Beauty» y el «Moral Sense» como su mayor originalidad, lo que ubicaba a la fórmula de la belleza en un marco más conservador, sin que se cayera en la cuenta de que la interpretación probabilística introducida en la Sección V era bastante vanguardista. Esto hizo que Dickie pusiera en duda el interés de la diferenciación entre el placer simple de los sentidos externos y el placer complejo del sentido de la belleza, así como que excluyera todo valor cognitivo del sentido de la belleza, que sería una facultad afectiva ${ }^{70}$, lo que se basaba en la inmediatez que Hutcheson concedió a nuestra vivencia de la belleza ${ }^{71}$. Dickie, con todo, concluyó que el discurso de Hutcheson fue ambiguo, pues con el término «belleza»se refirió tanto a la experiencia del sujeto como a las cualidades del objeto, lo que era problemático ${ }^{72}$. Así que su interpretación (la de Dickie) tuvo que decantarse por una opción u otra, eligiendo la más fértil históricamente, a saber, la experiencia subjetiva y el desinterés.

Si prestamos suficiente atención a la Sección V el planteamiento de Hutcheson emerge de nuevo con toda su coherente complejidad, según la cual sentimos placer ante la unión de uniformidad y variedad porque comprendemos su improbabilidad y, por lo tanto, su naturaleza diseñada. Cuando vemos, por ejemplo, un edificio, ordenado y pautado, surgir de una masa de rocas, aparentemente caótica, comprendemos la improbabilidad de que su ritmo sea producto de la roca, es decir, sabemos que ha sido diseñado. Que nuestra respuesta sea inmediata no anula este planteamiento, de la misma manera que no negamos el valor cognitivo del lenguaje porque usamos sus complejas reglas sin pensar explícita y conscientemente en ellas. Más que de inmediatez habría que hablar de «inmediatización ${ }^{73}$. Si aceptamos esto, la división de la historia de la estética en objetivismos y subjetivismos no es tan evidente, no al menos en el caso de Hutcheson, que calificaríamos más bien de «interaccionista» puro $^{74}$.

Quizá el argumento fundamental cuando se concede más importancia al sentido de la belleza que a la uniformidad en la variedad sea la dependencia de Hutcheson

${ }_{69}$ Con el que se podrían conectar, al menos, las tres primeras teorías de la belleza enumeradas por Scruton, R., Beauty, p. 5.

70 Dickie, G., El siglo del gusto, pp. 27 y 30-31.

71 Hutcheson, F., An Inquiry, p. 25; Dickie, George, El siglo del gusto, p. 32.

72 Dickie, G., El siglo del gusto, p. 34.

73 Una visión matizada con componentes racionales de la inmediatez de la experiencia estética, de una manera compatible con la que proponemos aquí, en GLAUSER, R., "The Experience of Absolute Beauty in Hutcheson: Perception, Reason and Pleasure», Journal of Scottish Thought, 7 (2016), 52-80, especialmente pp. 70-80.

74 Véase Matthews, P. M., "Hutcheson on the Idea of Beauty», en: Journal of the History of Philosophy, 36/2 (1998), 233-259; y Michael, E., «Francis Hutcheson on Aesthetic Perception and Aesthetic Pleasure», British Journal of Aesthetics, 24/3 (1984), 241-255. 
con respecto a Locke ${ }^{75}$, algo innegable, aunque no simple, al menos si atendemos a la propia epistemología de Hutcheson, tal y como aparece en su A Compend of Logic:

Ideas are divided into sensations, imaginations, and pure intellections.

Sensation is twofold, external and internal. External sensation is «the perception of a corporeal thing impacting the organs of the body».

Imagination is «the idea of a corporeal thing which is not impacting the body».

A pure intellection is «any idea which is not reached or grasped by any of the bodily senses». By intellection we not only discern things which are different from body as their modes, but we also attain more accurate ideas of numbers and of shapes which have several parts tan those which the senses provide ${ }^{76}$.

Tras ello continúa diferenciando ideas primarias y secundarias según el modelo lockeano y, según el mismo, introduciendo la reflexión, situando en ella las acciones, pasiones, etc., concluyendo que todas las ideas surgen a partir del sentido externo o de la reflexión, aunque la idea de número, esencial tanto en la fórmula de la belleza como en la teoría de la probabilidad, es percibida, según Hutcheson, a la vez por los sentidos externo e interno ${ }^{77}$.

Hemos recurrido a este texto por su tono condensado y brutal. Si en An Inquiring decía que las ideas «are rais'd in the Mind upon the presence of external Objects» ${ }^{78}$, aquí afirmaba que el objeto «impacts» sobre los órganos del cuerpo, lo cual muestra que detrás todo esto (también de Locke) estaba la famosa Ley III de los «Axiomas o leyes del movimiento» de Newton ${ }^{79}$. Este deseo de extender el exitoso modelo de Newton a las ciencias humanas, en este caso a la psicología, enraíza al subjetivismo en un esquema más amplio, de naturaleza interactiva, pues el objeto impacta sobre los sentidos del sujeto (como si se tratara de dos bolas de billar) y la reacción de estos define la sensación externa, producto de dicho encuentro.

Que esto presupone alguna forma de teoría corpuscular (en lo que no profundizaremos) queda claro a partir de la explicación que Hutcheson proporcionó de las sensaciones del gusto como el efecto de «the Forms of the small Bodys, or their Motions ${ }^{80}$. El criterio que siguió para diferenciar entre cualidades primarias

75 El estudio clásico es el Capítulo XV de KIvy, P., The Seventh Sense, pp. 283-300.

76 Hutcheson, F., Logic, Metaphysics, and the Natural Sociability of Mankind, pp. 11-12.

77 Hutcheson, F., Logic, Metaphysics, and the Natural Sociability of Mankind, p. 12. Véase Ablondi, F., «Hutcheson, Perception, and the Sceptic's Challenge», British Journal for the History of Philosophy, 20/2 (2012), 269-281; Ablondi se preguntaba por qué Hutcheson no se dio por enterado de las críticas de Berkeley y Hume a la epistemología de Locke, explicándolo por el propio contexto de la teología del diseño del autor (p. 281).

78 Hutcheson, F., An Inquiry, p. 19.

79 Newton, I., Principios matemáticos de la filosofía natural (1687), Madrid, Tecnos, 1987, p. 42. También, seguramente, las hipótesis sobre la percepción que presentó Newton en su Óptica. Véase Newton, I., Opticks (1704), Nueva York, Prometheus Books, 2003, pp. 346, 370 y 385-386 (hay traducción castellana: Óptica, Madrid, Alfaguara, 1977).

80 Hutcheson, F., An Inquiry, 35. Casi todos los científicos de finales del siglo XVII (incluido Newton, sobre todo en su Opticks) estaban de acuerdo en que la materia se componía de partículas, corpúsculos o átomos pequeños y discretos, con cuyo tamaño, forma y movimientos se podrían explicar las propiedades físicas y químicas de la materia. Véase Boss, M., «Newton's Chemical Papers», en Cohen, I. B., Isaac Newton's Papers and Letters on 
y secundarias era el aceptado a partir de Locke: las primarias (como las formas geométricas) se captaban con más de un sentido externo; las secundarias (como el color) con uno sólo. Hutcheson afirmaba, además, que las ideas primarias podían producir cualidades secundarias que no tenían equivalentes en los cuerpos mismos $^{81}$. Estas eran la reacción frente a la acción de los cuerpos externos, algo subjetivo generado a partir del «choque» objetivo producido en la realidad física. La única forma de reconstruir hipotéticamente la naturaleza del cuerpo que impactaba contra nosotros era mediante la coincidencia referencial de al menos dos sentidos, una suerte de triangulación sensorial, como resultado de la cual las ideas primarias eran "extension, figure, position, motion and rest ${ }^{82}$, las propiedades más básicas de los átomos desde la Antigüedad. Hutcheson podía aceptar esto porque no le preocupaba la estructura del sistema atomista sino su autonomía genética, es decir, su capacidad involuntaria de producir un orden a partir de del caos:

$[\ldots]$ we can not conceive that there is in matter either a natural or a necessary ability to move, but all the ability that it has would have been imparted by force of an intelligent nature. Even suppose that matter moved in some way or had the power of moving, but had no design or foresight, there could never have come from that the magnificent order we see in the world ${ }^{83}$.

Aunque era posible que la materia la definan las propiedades de las cualidades primarias, el cálculo de probabilidades aconsejaba no esperar del choque azaroso de tales partículas la emergencia de un orden recurrente. Era interesante mantener la estructura del atomismo porque la inmensa improbabilidad de su faceta genética demostraba la necesidad de un diseñador inteligente, puesto que era evidente que el mundo consistía en uniformidad más variedad en procesos de adaptación.

Esto nos lleva al primer parágrafo de la Sección $\mathrm{V}^{84}$, cuyo potencial ahora se comprenderá mejor. Hutheson utilizó la matemática de la probabilidad, el argumento del diseño y la hipótesis corpuscular para argumentar la coincidencia del sujeto con el objeto, requisito de su teoría relacional de la belleza, que sin duda quería asegurar. Primero propuso la posible aleatoriedad de la relación, es decir, que «the Constitution of our Sense so as to approve Uniformity, is merely arbitrary», de manera que «there are an infinity of Tastes or Relishes of Beauty possible»y, por lo tanto, «Other Minds may be so fram'd as to receive no Pleasure from Uniformity». Si todo es cuestión de azar, pues, podría haber innumerables subjetividades y objetividades no coincidentes, con lo que el placer (que es el resultado de dicha coincidencia) sería muy raro. La conclusión a la que llegó Hutcheson desde tales supuestos fue puramente probabilística, desarrollándose en dos fases. Primera:

Natural Philosophy, pp. 241-248, especialmente p. 244. El caso modélico de esta tendencia fue Robert Boyle, con su obra El químico escéptico (1661), cuyo ideario básico se fijaba en las dos Proposiciones de la Primera Parte (Boyle, R.: El químico escéptico. Barcelona, Crítica, 2012, pp. 60-62). Con respecto a la conexión de estos planteamientos con el problema de la relación entre el pensamiento y la materia en el siglo XVIII británico, véase YolTon, J. W., Thinking Matter. Materialism in Eighteenth-Century Britain, Oxford, Basil Blackwell, 1984, pp. 14-28 y 90-106.

81 Hutcheson, F., Logic, Metaphysics, and the Natural Sociability of Mankind, p. 12.

82 Hutcheson, F., Logic, Metaphysics, and the Natural Sociability of Mankind, p. 12.

83 Hutcheson, F., Logic, Metaphysics, and the Natural Sociability of Mankind, p. 160

84 Lo que sigue se basa en Hutcheson, F., An Inquiry, pp. 46-47. 
«[...] it would be impossible to throw together fifty or a hundred Pebbles, which should not make an agreeable Habitation for some Animal or other, and appear beautiful to it». Este ejemplo estadístico (lanzar algo a ver que sale) hacía que la aleatoriedad pareciera aceptable, pues para cualquier objeto podría haber un sujeto que gozara con su encuentro, fuera cual fuera su forma. Pero, segunda fase: a priori el encuentro sería tan improbable como sacar al mismo tiempo de dos bolsas con millones de bolas numeradas dos que tuvieran el mismo número, lo que explica la conclusión de Hutcheson:

But then, as there are an Infinity of Forms possible into which any System may be reduc'd, an Infinity of Places in which Animals may be situated, and an Infinity of Relishes or Senses in these Animals is suppos'd possible; that in the immense Spaces any one Animal should by Chance be plac'd in a System agreeable to its Taste, must be improbable as infinite to one at least.

Hutcheson tampoco estaba interesado aquí en negar la estructura corpuscular de la realidad porque le resultaba útil de nuevo para una prueba negativa adicional de Fitness. El azar como fuerza genética autónoma haría tanto del sujeto como del objeto compuestos aleatorios de partículas, siendo casi imposible que del choque aleatorio de cada uno de esos infinitesimales surgiera concordancia, es decir, experiencia placentera de la belleza. Mucho más improbable sería la recurrencia frecuente de la experiencia de la belleza que nos muestra nuestra vida cotidiana, lo que nos lleva al inicio y al final del parágrafo que analizamos. Comenzaba con esta consideración:

There seems to be no necessary Connection of our pleasing Ideas of Beauty with the Uniformity or Regularity of the Objects, from the Nature of things, antecedent to some Constitution of the Author of our Nature, which has made such Forms pleasant to $\mathrm{us}^{85}$.

Después de lo que hemos visto esto era bastante coherente. Siempre cabría la probabilidad azarosa de un encuentro de cualquier objeto con cualquier sujeto que generase una experiencia placentera de belleza, pero sería altamente inverosímil su recurrencia cotidiana, algo prácticamente impensable a priori, como expuso Hutcheson al final del parágrafo:

And much more unreasonable is it to expect from Chance, that a multitude of Animals agreeing in their Sense of Beauty should obtain agreeable Places ${ }^{86}$.

Para Hutcheson era evidente que dicha concordancia se producía, lo que le condujo a pensar que los dados de la partida estaban trucados, es decir, que había algo más que azar. La experiencia de la belleza era, por lo tanto, el ejemplo más evidente de que el mundo y nuestra posición en él habían sido proyectados por un ser inteligente, poderoso y benigno. Esta conclusión dependía del planteamiento probabilístico del primer parágrafo de la Sección V, aunque fue más desarrollada en las Secciones VI y VII del Tratado I, que giran en torno a la idea de que nuestro sentido de la belleza fue diseñado para proporcionarnos placer a todos ${ }^{87}$. Ambas

\footnotetext{
85 Hutcheson, F., An Inquiry, p. 46.

86 Hutcheson, F., An Inquiry, p. 47.

87 Hutcheson, F., An Inquiry, p. 62.
} 
secciones se basaban en la existencia del diseñador omnipotente, presente en toda su estética y clave para comprender su rechazo del relativismo, pues la diferencia de los gustos, debida a la asociación de ideas, la educación y el ejemplo, no invalidaba la fórmula de la belleza sino, simplemente, modificaba el contenido de que se la dotaba, concurriendo aquélla constantemente con el cambio de los estilos y las modas, como mostraba la experiencia ${ }^{88}$. La arquitectura que gustaba a un godo, por ejemplo, era diferente (Hutcheson creía que inferior, aunque eso sí que es secundario) de la que gustaba a un griego, pero el primero también uniformaba lo diverso al construir y valorar sus edificios. Esa misma uniformidad de respuestas entre la variedad de estilos, ejemplificada con el objeto cultural diseñado por el ser humano, era extensible a la respuesta ante la realidad natural, que no fue proyecto suyo ${ }^{89}$.

Si interpretamos a Hutcheson en términos psicologistas y nos centramos en la dimensión experiencial de la belleza como algo anclado en el sujeto y que nada afirma del objeto, nos topamos con el problema del relativismo del gusto y la imposibilidad del discurso estético. La fórmula de la uniformidad en la diversidad se puede interpretar como un recurso para superar dicho relativismo ${ }^{90}$. Según Korsmeyer, la defensa del desinterés estético y el uso de dicha fórmula evitaban el relativismo y aseguraban el terreno para el "Moral Sense " " , aunque nosotros creemos que así no se puede dimensionar adecuadamente la tarea de Hutcheson (tampoco siguiendo la vía contraria de reducción de la ética a la estética ${ }^{92}$ ), que fue más profunda y compleja. Hutcheson no afrontó un problema antropológico centrado en el sujeto empírico y su comportamiento moral, sino otro ontológico conectado con la visión global de la realidad que el nuevo paradigma científico estaba definiendo desde el siglo XVII. La tarea consistía en aunar con coherencia la realidad matemática mostrada en los Principia de Newton con una química corpuscular que tendía hacia el azar, como la de Boyle en El químico escéptico. La integración de esos dos modelos no se había producido y una manera de afrontarla era a través de la hipótesis de un diseñador inteligente. Hutcheson usó la matemática de la probabilidad de su época para asegurar dicha hipótesis. El problema del relativismo debe situarse en

88 Hutcheson, F., An Inquiry, p. 63. Para lo que sigue, pp. 63-66.

89 Véase Korsmeyer, C., "The Two Beauties: A Perspective on Hutcheson's Aesthetics», The Journal of Aesthetics and Art Criticism, 38/2 (1979), pp. 145-151, quien afirmó que la diferenciación entre belleza absoluta y relativa encubría el colapso de la teoría del arte en la teoría estética (p. 149). Se trata de algo dudoso, dado que también la arquitectura, la jardinería, la ebanistería y la música, por ejemplo, son artes de las que no se puede predicar una naturaleza imitativa, mientras que no se pueden negar los valores constructivos de la pintura o de la escultura. Hutcheson usó las artes como un modelo para el argumento del diseño, según una analogía que proviene de Cicerón y de Shaftesbury. Nosotros sugerimos que tal vez sucede lo contrario, es decir, que la teoría del arte se extiende a la totalidad de la estética.

90 Así lo planteó de nuevo Carolyn WiLKer Korsmeyer, "Relativism and Hutcheson's Aesthetic Theory», Journal of the History of Ideas, 36/2 (1975), 319-330.

91 Korsmeyer, C., «The Two Beauties: A Perspective on Hutcheson's Aesthetics», p. 146. Una interpretación similar, aunque en el campo de la ética, en EAgLETon, T., «Homage to Francis Hutcheson», en: Heathcliff and the Great Hunger, Londres, Verso, 1995, p. 109.

92 Como propone, por ejemplo, BROADIE, A., «Francis Hutcheson, George Turnbull and the Intersection of Aesthetics and Morals», en: Journal of Scottish Thought, 7 (2016), 1-15 (especialmente pp. 14-15). 
este contexto de más amplio alcance, donde lo que más importaba no era el acuerdo entre sujetos sino la consistencia de la realidad, incluida la humana ${ }^{93}$. Lo que preocupaba a Hutcheson del sujeto empírico era su vinculación con el mundo que Dios había diseñado, pensando que quedaba garantizada cuando respondíamos a la belleza de una manera inmediata, impremeditada y desinteresada. Lo que, de una manera sucinta e incompleta, dijo Taylor de este contexto histórico es aplicable a Hutcheson más que a nadie:

These Scottish philosophers began with natural theology, worked on to philosophy and logic, and thence proceeded to political economy and jurisprudence ${ }^{94}$.

Todo esto resulta más difícil de aceptar si no se reconoce la relevancia de la Sección V, la cual permite, además, integrar mejor la estética de Hutcheson con el resto de su obra ${ }^{95}$. Sus reflexiones sobre la belleza se suelen analizar como anticipo de la estética posterior o como preparación de su teoría moral, pero teniendo en cuenta la Sección V resultan ser algo más. La uniformidad en la diversidad adquiere todo su sentido en el contexto del cálculo de probabilidades y el uso que Hutcheson hizo de él en la prueba de la existencia del Dios diseñador. Su estética, por lo tanto, no fue sólo una preparación de su ética, sino también de su teología natural. Si consideramos, como hizo Dickie, poco interesante la Sección V, la afirmación de éste es correcta: «las conclusiones teológicas de Hutcheson no tienen como fin respaldar su teoría del gusto; solamente la completan ${ }^{96}$; pero si no la consideramos así lo que resulta es la profunda implicación de la estética de Hutcheson en su teología natural, lo cual aconseja que se la ubique de otra manera en su pensamiento, no como preparación de su ética sino como fundamento de la totalidad de su sistema.

\section{CONCLUSIÓN}

En el desarrollo del pensamiento de Hutcheson posterior a 1725 el argumento del diseño apareció constantemente y en todos los niveles de análisis. El diseño explicaba la realidad natural y cómo respondemos ante ella, pero también nuestra estructura como sujetos y la articulación de la sociedad en sus dimensiones moral,

93 Muy acertadamente, Remo BoDEI lo define como una «teodicea estética» en La forma de lo bello, Madrid, Antonio Machado, 2008, p. 39.

94 TAYlOR, W. L., Francis Hutcheson and David Hume as Predecessors of Adam Smith, Durham/North Carolina, Duke University Press, 1965, p. 9.

95 Véase, sin embargo, Cifuentes Quiñonez, L. A., "La Modernidad Estética en Una Investigación sobre el Origen de Nuestra Idea de Belleza», Universitas Philosophica, 28 (1997), 2947; aunque fue un estudio equilibrado de la estética de Hutcheson, donde tanto los aspectos intelectuales como la importancia de la probabilidad para el argumento del diseño fueron tratados correctamente (pero de manera insuficiente), interpreta el papel histórico de la obra de Hutcheson según el modelo habitual, como pionero de la autonomía estética basada en el desinterés. Es decir, valioso por lo que supuso de anticipación de Hume y Kant, según la pauta establecida por Stolnitz.

96 Dickie, G., El siglo del gusto, p. 52. 
política, judicial o económica ${ }^{97}$. Dada la importancia de la belleza en dicho argumento, la estética, entendida en términos relacionales, no fue sólo una dimensión instrumental y aislable en este pensamiento, sino la condición de posibilidad de su visión de la realidad, articulada en torno a la Sección V.

Para Hutcheson, nuestra respuesta placentera ante la conexión de la uniformidad con la variedad era una parte del plan del gran Autor de la naturaleza ${ }^{98}$, algo que no dependía de nosotros pero que tampoco era fruto del azar, sino de la elección de aquel Agente supremo que constituyó nuestros sentidos, incluidos los internos ${ }^{99}$. Tratando de desentrañar los motivos que movieron a Dios a trucar así los dados, Hutcheson recurrió a una explicación epistémica y no estética, moral, política o religiosa: puesto que somos seres limitados, esta construcción nos permitía un acceso adecuado al conocimiento de la realidad física y a la planificación de nuestras acciones en este mundo. Así concluyó la primera edición del Tratado I de An Inquiry:

The Universe must be govern'd not by particular Wills, but by general Laws, upon which we can found our Expectations, and Project our Schemes of Action ${ }^{100}$.

Esta interpretación del pensamiento de Hutcheson, y del papel que la estética juega en él, permite proponer una aproximación diferente al influjo histórico de su obra, aunque aquí no podemos desarrollarla. Pensemos, como ejemplo y a modo de conclusión, en su alumno Adam Smith. Hoy día es un tema intenso de debate el newtonianismo de Smith, la medida en la que éste construyó su obra a partir del modelo de los Principia Mathematica. Este debate se basa sobre todo en el estudio explícito que Smith hizo de la posición de Newton en la historia de la astronomía ${ }^{101}$. Smith desarrolló una historia psicológica y social de la astronomía que se basaba en las exigencias de la imaginación, lo que ha llevado a rastrear sus conexiones con el Tratado de la naturaleza humana de su amigo Hume ${ }^{102}$. Sin embargo, el estudio que hizo Smith de la obra astronómica de Newton y su papel en la historia del pensamiento se basaba en un criterio evaluativo que, aunque se remitía a la imaginación, se complacía sobre todo al unificar el mayor número posible de hechos

97 Sería tema para otro artículo, pero por poner algunos ejemplos (que distan de agotar los posibles): Hutcheson, Francis, An Inquiry (Treatise II), p. 197; An Essay on the Nature and Conduct of the Passions and Afections, with Illustrations on the Moral Sense (1728), Indianapolis, Liberty Fund, 2002, pp. 8, 42-43, 116-120, 132; Philosophiae Moralis Institutio Compendiaria, with A Short Introduction to Moral Philosophy, Indianapolis, Liberty Fund, 2007, pp. 24-25, 76-97; Logic, Metaphysics, and the Natural Sociability of Mankind, pp. 81, 97, 114-115, 148-149, 174-175, 177-178, 192, 195-196 (prácticamente toda la parte A Synopsis of Metaphysics es un desarrollo del argumento del diseño en sus varias facetas); A System of Moral Philosophy, Volume 1(1755), Cambridge, Cambridge University Press, 2014, pp. 168-208.

98 Hutcheson, F., An Inquiry, p. 78.

99 Hutcheson, F., An Inquiry, p. 80.

100 Hutcheson, F., An Inquiry, p. 82.

101 Sмiтн, A., The Principles which Lead and Direct Philosophical Enquiries; Illustrated by the History of Astronomy, en Essays on Philosophical Subjects, pp. 31-105, sobre Newton pp. 97-105.

102 Schliesser, E., «Copernican Revolutions Revisited in Adam Smith by way of David Hume», en: Revista Empresa y Humanismo, XIII (2010), 213-248. 
observados a través del menor número posible de principios explicativos ${ }^{103}$. Dicho de otra manera, la imaginación científica operaba según el principio de la uniformidad en la variedad, el cual provenía de la definición de la belleza de su maestro Hutcheson, quien usó en ocasiones el sistema de Newton como ejemplo de la belleza de los teoremas ${ }^{104}$.

Por otra parte, en su historia de la física, que seguía y complementaba la de la astronomía, Smith se refirió a la idea de

[...] an universal mind, of a God of all, who originally formed the whole, and who governs the whole by general laws, directed to the conservation and prosperity of the whole ${ }^{105}$.

Este Dios diseñador, instaurador de un orden inteligente, cuyo reconocimiento para el pretendidamente escéptico Hume fue la forma más aceptable de religiosidad $^{106}$, provenía de Hutcheson, como la definición del concepto de belleza que sirvió de criterio para su historia de la astronomía ${ }^{107}$. A la luz del análisis que hemos desarrollado en lo que antecede, el influjo de Hutcheson se podría mostrar mayor de lo que hasta ahora parecía, por ejemplo, sobre las obras más importantes de Adam Smith (cuyo fundamento teológico, mediante la metáfora de la «mano invisible», es también hoy un campo intenso de estudio ${ }^{108}$ ) y, a través de él, sobre nosotros.

Hutcheson pensaba que nuestra imaginación (como la de los astrónomos de Adam Smith) necesitaba el orden porque, a priori, todo indicaba que podría ser el caos. Por eso le resultaba necesaria la existencia del Dios diseñador, que se daba a

103 Smith, A., Essays on Philosophical Subjects, pp. 103-105.

104 Hutcheson, F.s, An Inquiry, pp. 38-39. El tema de la belleza de la ciencia proviene seguramente de J. P. de Crousaz, Traité du Beau, Capítulo 9, pp. 175-257, donde habla, entre otras cosas, de la belleza de la física y la matemática y su aportación a la «Théologie naturelle» (p. 178). El primer contacto de Smith con el tema sería a través de Hutcheson y posiblemente de éste provendría la conexión de Smith con el Traité de Crousaz, del que poseía un ejemplar en su biblioteca (Mizuta, Hiroshi, Adam Smith's Library, Cambridge, Cambridge University Press, 1967, p. 16).

105 Sмітн, A., Essays on Philosophical Subjects, p. 113.

106 Hume, D., Historia natural de la religión (1757), Madrid, Tecnos, 2010, pp. 11-12. Esto debería leerse en conexión con el ambiguo cierre de sus Diálogos sobre la religión natural (1779), Madrid, Tecnos, 1994, p. 188: «no puedo sino pensar que los principios defendidos por Filón son más probables que los de Demea, pero que los de Cleantes se acercan aún más a la verdad». Hay una reciente interpretación que matiza y reduce el escepticismo religioso de Hume: Willis, A. C., Toward a Humean True Religion: Genuine Theism, Moderate Hope, and Practical Morality, Pennsylvania State University Press, 2014, especialmente pp. 44-86.

107 Es bastante penoso que casi todas las historias contemporáneas de la teología natural, bastante valiosas por otra parte, tratan por extenso a Hume pero ignoran por completo a Hutcheson, incluso cuando incluyen partes dedicadas a las conexiones de la estética con la teología natural (sirvan como ejemplo Craig, William Lane y J.P. Moreland -eds., The Blackwell Companion to Natural Theology, Malden, Blackwell, 2012 o Manning, Russell Re -ed., The Oxford Handbook of Natural Theology, Oxford, Oxford University Press, 2013). Sin embargo, el referente inmediato de los análisis de Hume fue, sin duda, Hutcheson. Aquél no puede entenderse sin éste.

108 Véase, por ejemplo, Osuington, P. (ed.), Adam Smith as Theologian, New York, Routledge, 2011. 
conocer en primer lugar en nuestra experiencia de la belleza. El deleite inmediato e impremeditado ante la uniformidad en la diversidad era una estrategia de captación que nos anclaba en una realidad digna de ser conocida en todas sus dimensiones. La consideración ordenada del mundo era primero una intuición básica de naturaleza estética, inmediata pero compleja (pues implicaba el reconocimiento de la uniformidad en la diversidad, la improbabilidad y las innumerables adaptaciones); esta intuición proporcionaba la chispa que daba lugar a los programas científicos y los proyectos de ordenación de la realidad social, los cuales profundizaban epistémicamente en y ponían en práctica dicha intuición, la más básica del ser humano. Sin la experiencia previa de la belleza no hubieran sido posible los Principia de Newton, pero tampoco La Riqueza de las Naciones de su alumno Adam Smith. Se trata de una manera de proceder que puede seguir siendo vinculante para nosotros, posdarwinianos que, abandonando el mundo a su suerte, dudamos que haya sido diseñado. Basta con que nos parezca hermoso ${ }^{109}$.

Universidad de Sevilla

JoRGE LÓPEZ LLORET

Departamento de Estética e Historia de la Filosofía.

lopezlloret@us.es

[Artículo aprobado para publicar en enero de 2018]

109 Véanse los dos primeros capítulos de Dawkins, R., The Blind Watchmaker (1986), Londres, Penguin, 2006 (hay traducción castellana: El relojero ciego, Barcelona, Tusquets, 2015), que tratan, precisamente, de la explicación de lo muy improbable (Capítulo 1, pp. 1-20), es decir, el buen diseño (Capítulo 2, pp. 21-42), dedicando el resto del libro a mostrar que la hipótesis del diseñador no resulta necesaria. Pese a todo, en otra obra posterior (1998) sigue aceptando la belleza de la ciencia, hallándose entre sus ejemplos el que proporciona el título al libro, a saber, la admirable conexión de unidad de principio y diversidad de efectos en el análisis de la composición y comportamiento de la luz por Newton; Dawkins, R., Unweaving the Rainbow, Londres, Penguin, 2006, pp. 63-82 (en castellano: Destejiendo el arco iris, Barcelona, Tusquets, 2012). Si Hutcheson hubiera sido ateo y, además, dispuesto de la hipótesis de un tiempo inmenso, seguramente habría llegado a conclusiones similares. 